\title{
A CHARACTERIZATION OF WEAK PSEUDOCONVEXITY
}

\author{
AKIRA SAKAI \\ (Communicated by Irwin Kra)
}

\begin{abstract}
It is proved that a smooth domain $D$ of $\mathbf{C}^{n}$ is weakly pseudoconvex, if, for every strongly pseudoconvex domain $D^{\prime}$ with $D \cap D^{\prime}=\varnothing$ and $E=\bar{D} \cap \bar{D}^{\prime} \neq \varnothing, E$ is totally real.
\end{abstract}

Let $D$ be a bounded domain of $\mathbf{C}^{n}$ with $C^{2}$ boundary and $p$ a point of $\partial D . D$ is said to be weakly (or strongly) pseudoconvex at $p$ if, for every $C^{2}$ function $\rho$ on an open neighborhood $U$ of $p$ in $C^{n}$ such that $D \cap U=$ $\{z \in U: \rho(z)<0\}$ and $d \rho(p) \neq 0$, the Levi form

$$
L[\rho ; \zeta](p)=\sum_{j k} \frac{\partial^{2} \rho}{\partial z_{j} \partial \bar{z}_{k}}(p) \zeta_{j} \bar{\zeta}_{k}
$$

is nonnegative (or positive, resp.) for any nonzero vector $\zeta$ with $\sum_{j} \frac{\partial \rho}{\partial z_{j}}(p) \zeta_{j}=$ 0 . We say that $D$ is weakly (or strongly) pseudoconvex if $D$ is weakly (or strongly resp.) pseudoconvex at every point of $\partial D$. A subset $T$ is called a totally real set, if it is the zero set of a nonnegative $C^{2}$ strongly plurisubharmonic function on an open neighborhood of $T$. A $C^{1}$ submanifold $M$ is totally real if and only if it has no nonzero complex tangent vectors.

It is known that if $D$ is a weakly pseudoconvex domain and $D^{\prime}$ is a strongly pseudoconvex domain with $D \cap D^{\prime}=\varnothing$ then $T=\bar{D} \cap \bar{D}^{\prime}$ is a totally real set, provided $T$ is nonempty (see [1] and [2]). As a converse of this fact, we prove the following theorem.

Theorem. Let $D$ be a domain with $C^{3}$ boundary. If, for every strongly pseudoconvex domain $D^{\prime}$ such that $D \cap D^{\prime}=\varnothing$ and $M=\bar{D} \cap \bar{D}^{\prime}$ is a real $C^{1}$ submanifold, $M$ is totally real, then $D$ is weakly pseudoconvex.

Proof. We assume that $n>1$, since, in the case $n=1$, all domains with $C^{2}$ boundaries are strongly pseudoconvex. Let $z=\left(z_{1}, \ldots, z_{n}\right), z_{j}=x_{j}+i y_{j}$, $j=1, \ldots, n$, denote the complex coordinates of $\mathbf{C}^{n}$. Suppose that $D$ is not

Received by the editors January 29, 1988.

1980 Mathematics Subject Classification (1985 Revision). Primary 32F15.

Key words and phrases. Weakly and strongly pseudoconvex domain, Levi form, totally real. 
weakly pseudoconvex at $p$. After a suitable holomorphic change of coordinates, we can assume that $p$ is the origin and that $D$ is locally written as

$$
y_{n}<\phi\left(z_{1}, z^{\prime}, x_{n}\right), \quad z^{\prime}=\left(z_{2}, \ldots, z_{n-1}\right),
$$

where $\phi$ is a $C^{3}$ function on an open neighborhood $N$ of the origin in the hypersurface $y_{n}=0$ whose derivatives of the first order at the origin are all zero and which satisfies

$$
\frac{\partial^{2} \phi}{\partial z_{1} \partial \bar{z}_{1}}(0,0,0)>0
$$

By expanding $\phi$ in a Taylor series, we have

$$
\begin{aligned}
\phi\left(z_{1}, z^{\prime}, x_{n}\right)=\phi\left(z_{1}, 0,0\right)+\sum_{j=2}^{n-1} \frac{\partial \phi}{\partial z_{j}}\left(z_{1}, 0,0\right) z_{j}+\sum_{j=2}^{n-1} \frac{\partial \phi}{\partial \bar{z}_{j}}\left(z_{1}, 0,0\right) \bar{z}_{j} \\
+\frac{\partial \phi}{\partial x_{n}}\left(z_{1}, 0,0\right) x_{n}+O\left(\sum_{j=2}^{n-1}\left|z_{j}\right|^{2}+x_{n}^{2}\right) .
\end{aligned}
$$

We define the function

$$
\begin{aligned}
\psi\left(z_{1}, z^{\prime}, x_{n}\right)=\phi\left(z_{1}, 0,0\right)+\sum_{j=2}^{n-1} & \frac{\partial \phi}{\partial z_{j}}\left(z_{1}, 0,0\right) z_{j}+\sum_{j=2}^{n-1} \frac{\partial \phi}{\partial \bar{z}_{j}}\left(z_{1}, 0,0\right) \bar{z}_{j} \\
& +\frac{\partial \phi}{\partial x_{n}}\left(z_{1}, 0,0\right) x_{n}+c\left(\sum_{j=2}^{n-1}\left|z_{j}\right|^{2}+x_{n}^{2}\right),
\end{aligned}
$$

where $c$ is a positive constant. If $c$ is sufficiently large, then we have $\psi \geq$ $\phi$. The equality holds just when $z_{2}=\cdots=z_{n-1}=x_{n}=0$. We put $\sigma=$ $\psi\left(z_{1}, z^{\prime}, x_{n}\right)-y_{n}$ and $D_{1}=\{z: \sigma(z)<0\}$. Then the intersection $\bar{D} \cap \bar{D}_{1}$ is the manifold

$$
M=\left\{z: z_{2}=\cdots=z_{n-1}=x_{n}=0, \phi\left(z_{1}, z^{\prime}, x_{n}\right)=y_{n}\right\} .
$$

For every vector $\zeta$, we have

$$
\begin{aligned}
L[\sigma, \zeta](0)= & \frac{\partial^{2} \phi}{\partial z_{1} \partial \bar{z}_{1}}(0,0,0)\left|\zeta_{1}\right|^{2}+2 \operatorname{Re}\left[\sum_{j=2}^{n-1} \frac{\partial^{2} \phi}{\partial z_{1} \partial \bar{z}_{j}}(0,0,0) \zeta_{1} \bar{\zeta}_{j}\right] \\
& +\operatorname{Re}\left[\frac{\partial^{2} \phi}{\partial z_{1} \partial x_{n}}(0,0,0) \zeta_{1} \bar{\zeta}_{n}\right]+c\left[\sum_{j=2}^{n-1}\left|\zeta_{j}\right|^{2}+\frac{1}{4}\left|\zeta_{n}\right|^{2}\right] .
\end{aligned}
$$

We write the right member as $F(\zeta)+c G(\zeta)$. Then $F$ and $G$ are continuous on $\Gamma=\left\{\zeta \in C^{n}:|\zeta|=1\right\}$ and $G$ is nonnegative. When $G(\zeta)=0$, we have

$$
F(\zeta)=\frac{\partial^{2} \phi}{\partial z_{1} \partial \bar{z}_{1}}(0,0,0)\left|\zeta_{1}\right|^{2}>0
$$


Therefore, we can find a constant $c$ so that $L[\sigma ; \zeta](0)>0$ for every nonzero vector $\zeta$. Hence $\sigma$ is strongly plurisubharmonic in an open neighborhood of the origin.

Thus we can find an open neighborhood $V$ of the origin and a strongly pseudoconvex domain $D^{\prime}$ contained in $D_{1}$ such that $\partial D^{\prime} \cap V$ coincides with $\partial D_{1} \cap V$. The tangent space of the manifold $M \cap V$ at the origin is the $z_{1}$-plane and hence $M \cap V$ is not totally real. This proves the theorem.

We remark that the theorem is also valid for domains of complex manifolds, since all the arguments are quite local.

\section{REFERENCES}

1. Th. Duchamp and E. L. Stout, Maximum modulus sets, Ann. Inst. Fourier (Grenoble) 31 (1981), 37-69.

2. A. Sakai, The intersection of the closures of two disjoint strongly pseudoconvex domains, Math. Ann. 260 (1982), 117-118.

Department of Mathematical Sciences, College of Engineering, University of Osaka Prefecture, Sakai 591 Japan 